PROCEEDINGS OF THE

AMERICAN MATHEMATICAL SOCIETY

Volume 128, Number 2, Pages 573-581

S 0002-9939(99)05076-5

Article electronically published on July 6, 1999

\title{
INVERTIBILITY IN INFINITE-DIMENSIONAL SPACES
}

\author{
CHIA-CHUAN TSENG AND NGAI-CHING WONG
}

(Communicated by Dale Alspach)

\begin{abstract}
An interesting result of Doyle and Hocking states that a topological $n$-manifold is invertible if and only if it is a homeomorphic image of the $n$-sphere $S^{n}$. We shall prove that the sphere of any infinite-dimensional normed space is invertible. We shall also discuss the invertibility of other infinite-dimensional objects as well as an infinite-dimensional version of the Doyle-Hocking theorem.
\end{abstract}

\section{INTRODUCTION}

The most interesting application of invertibility in finite-dimensional spaces is the Doyle-Hocking characterization of the $n$-sphere $S^{n}$.

Theorem 1 (Doyle and Hocking [8]). A topological n-manifold is homeomorphic to $S^{n}$ if and only if it is invertible.

A (non-empty) topological space $X$ is said to be invertible [9] if for each proper open subset $U$ of $X$ there is a homeomorphism $T$ (called an inverting homeomorphism) of $X$ onto $X$ sending $X \backslash U$ into $U$. Recall that a subset $U$ of $X$ is proper if both $U$ and its complement $X \backslash U$ are not empty. It is clear that invertibility is a topological property, i.e. preserved by homeomorphisms. In many cases, we may expect that a topological property which holds locally in an arbitrary proper open subset $U$ of $X$ holds indeed globally in all of $X$. For examples, we have

Proposition $2([9,15,10,13,16])$. Let $U$ be a proper open subset of an invertible space $X$. If $U$ has any of the following properties, then $X$ also has the corresponding properties: (1) $T_{0}$, (2) $T_{1}$, (3) Hausdorff, (4) regular, (5) completely regular, (6) normal, (7) first countable, (8) second countable, (9) separable, (10) metrizable, (11) uniformizable, (12) compact, (13) pseudocompact, (14) extremally disconnected; unless $X$ is a two point space, the list also includes: (15) $T_{1}$ and connected, and (16) $T_{1}$ and path connected.

Recall that a topological space $X$ is locally compact if every point $x$ in $X$ has a compact neighborhood $U$, i.e. $x$ belongs to the interior of the compact subset $U$ of $X$. Since locally compact invertible spaces must be compact, the intervals

Received by the editors June 20, 1997 and, in revised form, April 14, 1998.

1991 Mathematics Subject Classification. Primary 46B20, 57N20, 57N50.

Key words and phrases. Invertible spaces, spheres, infinite-dimensional topology, infinitedimensional manifolds.

This work was partially supported by the National Science Council of Republic of China. Grant Number: NSC 83-0208-M-110-0171, 87-2115-M-110-002.

(C)1999 American Mathematical Society 
$(0,1),[0,1)$ and $(0,1]$, and the $n$-space $\mathbb{R}^{n}(n=1,2, \ldots)$ cannot be invertible. By a simple connectedness argument, one can see that the compact interval $[0,1]$ is not invertible, either. On the other hand, all finite-dimensional spheres $S^{n}(n=$ $1,2, \ldots)$, the set $\mathbb{Q}$ of all rational points of the real line $\mathbb{R}$, and the Cantor set are all invertible. Moreover, it is easy to show that a topological space $X$ is invertible if and only if for any proper closed subset $F$ and proper open subset $U$ of $X$ there is a homeomorphism of $X$ onto itself sending $F$ into $U$. Consequently, one can see that many fractal figures are invertible along the line of reasoning in [9], in which together with several continua the universal one-dimensional plane curve is proved to be invertible. It seems to us that invertibility may be a useful tool in studying fractal geometry. Finally, an interesting presentation of the theory of function spaces of invertible spaces can be found in [18].

This paper is devoted to an infinite-dimensional version of Theorem 1. In particular, we shall show

Theorem 3. The unit sphere of any normed space of finite or infinite dimension is invertible. Moreover, the inverting homeomorphisms $T$ can be chosen to have period 2, i.e. $T \circ T$ is the identity map of the sphere.

Conjecture 4. All infinite-dimensional invertible topological Hilbert manifolds are homeomorphic to the unit sphere of the underlying Hilbert space.

Recall that a topological space $X$ is called a (topological) manifold modeled on a topological vector space $E$ if there is an open cover of $X$ each member of which is homeomorphic to $E$. The following result of Torunczyk tells us that we may consider merely Hilbert manifolds (i.e. the case that the model space $E$ is a Hilbert space).

Theorem 5 (Toruńczyk $[19,20]$ ). All infinite-dimensional Fréchet (i.e. complete metrizable locally convex) spaces are homeomorphic to Hilbert spaces.

The invertibility of infinite-dimensional spheres and other convex objects will be verified in Section 2. Some approaches to solving Conjecture 4 will be presented in Section 3 .

\section{MAIn RESUlts}

Recall that a convex subset of a topological vector space is called a convex body if it has non-empty interior. Since the unit ball of a normed space is a bounded convex body, Theorem 3 follows from the following seemingly more general

Theorem 6. The (topological) boundary $S$ of any bounded convex body $V$ in any normed space $N$ is invertible. Moreover, the inverting homeomorphisms can be chosen to have period 2.

Proof. We may assume that $N$ is a real normed space of dimension greater than 1 . In fact, if the underlying field is complex, then we may consider the real normed space $N_{\mathbb{R}}$ instead. $N_{\mathbb{R}}$ is the vector space $N$ over the real field $\mathbb{R}$ equipped with the norm $\|\cdot\|_{\mathbb{R}}$, where $\|x\|_{\mathbb{R}}=\|x\|$ for all $x$ in $N$. It is plain that $(N, V)$ and $\left(N_{\mathbb{R}}, V\right)$ are homeomorphic as topological pairs. The case that $N$ is the one-dimensional line $\mathbb{R}$ is trivial. Moreover, we may assume that $V$ is open and contains 0 since the boundary of any convex body coincides with the boundary of its interior.

Recall that in the proof of the invertibility of finite-dimensional spheres $S^{n}$, one utilizes the stereographic projection of $S^{n} \backslash\{\infty\}$ onto $\mathbb{R}^{n}$ and the inversions of $\mathbb{R}^{n}$ 
with respect to circles. To achieve an infinite-dimensional version of these type of arguments, the first task for us is to replace $S$ with a homeomorphic image $S_{2}$ which looks "round" enough to have a stereographic projection onto a closed hyperplane of $N$. Then the inverting homeomorphisms will be obtained exactly the same way as in the finite-dimensional case.

Let $r$ be the gauge functional of the open convex set $V$, namely,

$$
r(x)=\inf \{\lambda>0: x \in \lambda V\}, \quad \forall x \in N .
$$

$r$ is a sublinear functional of $N$ since $V$ is convex. In other words, $r(x+y) \leq$ $r(x)+r(y)$ and $r(\lambda x)=\lambda r(x)$ for all $x, y$ in $N$ and $\lambda \geq 0$.

Claim 1. There is a constant $\alpha>1$ such that $\frac{1}{\alpha} U_{N} \subseteq V \subseteq \alpha U_{N}$; or equivalently,

$$
\frac{1}{\alpha} r(x) \leq\|x\| \leq \alpha r(x), \quad \forall x \in N,
$$

where $U_{N}=\{x \in N:\|x\| \leq 1\}$ is the closed unit ball of $N$.

In fact, the openness and boundedness of $V$ establish the inclusions for some constant $\alpha>1$. For the norm inequalities, we observe that, for any non-zero $x$ in $N, x /\|x\| \in U_{N} \subseteq \alpha V$ implies that $r(x /\|x\|) \leq \alpha$ or $r(x) \leq \alpha\|x\|$. Similarly, since $x / r(x)$ belongs to the closure of $V \subseteq \alpha U_{N}$, we have $\|x / r(x)\| \leq \alpha$ or $\|x\| \leq \alpha r(x)$, as asserted.

As a consequence of Claim 1, the family $\left\{B_{r, 1 / n}(x): n=1,2, \ldots\right\}$ is a local base at each $x$ in $N$ in the norm topology, where $B_{r, 1 / n}(x)=\{y \in N: r(y-x) \leq 1 / n\}$. It is easy to see that $S=\{x \in N: r(x)=1\}$. Fix an arbitrary $x_{0}$ in $S$ and let $f$ be a continuous (real) linear functional of $N$ supporting $V$ at $x_{0}$, i.e. $f(x) \leq f\left(x_{0}\right)=$ $1, \forall x \in V$. Write

$$
N=\mathbb{R} x_{0} \oplus \operatorname{Ker} f
$$

as a direct sum of the line $\mathbb{R} x_{0}$ in the direction of $x_{0}$ and the closed hyperplane $\operatorname{Ker} f=\{y \in X: f(y)=0\}$ determined by $f$. For each $x$ in $N$, write

$$
x=f(x) x_{0}+y_{x}
$$

for some (unique) $y_{x}$ in Ker $f$. Define another sublinear functional $r_{2}$ of $N$ by

$$
r_{2}(x)=\sqrt{f(x)^{2}+r\left(y_{x}\right)^{2}}, \quad \forall x \in N .
$$

Claim 2. There are positive constants $c$ and $d$ such that $c r_{2}(x) \leq r(x) \leq d r_{2}(x)$, $\forall x \in N$.

By the norm inequalities (1), we have

$$
|f(x)| \leq\|f\|\|x\| \leq \alpha\|f\| r(x)
$$

and

$$
\begin{aligned}
r\left(y_{x}\right) & =r\left(x-f(x) x_{0}\right) \leq \alpha\left\|x-f(x) x_{0}\right\| \leq \alpha\left(\|x\|+|f(x)|\left\|x_{0}\right\|\right) \\
& \leq \alpha^{2}\left(1+\|f\|\left\|x_{0}\right\|\right) r(x)
\end{aligned}
$$

for all $x$ in $N$. Consequently,

$$
r_{2}(x)^{2} \leq\left(\alpha^{2}\|f\|^{2}+\alpha^{4}\left(1+\|f\|\left\|x_{0}\right\|\right)^{2}\right) r(x)^{2}, \quad \forall x \in N .
$$

On the other hand,

$$
\begin{aligned}
r(x) & \leq r\left(f(x) x_{0}\right)+r\left(y_{x}\right) \leq \alpha|f(x)||| x_{0} \|+r\left(y_{x}\right) \leq \alpha^{2}|f(x)|+r\left(y_{x}\right) \\
& \leq \alpha^{2}\left(|f(x)|+r\left(y_{x}\right)\right),
\end{aligned}
$$


and hence

$$
r(x) \leq \sqrt{2} \alpha^{2} r_{2}(x)
$$

for all $x$ in $N$.

It follows from Claims 1 and 2 that the family $\left\{B_{r_{2}, 1 / n}(x): n=1,2, \ldots\right\}$ forms a local base at each $x$ in $N$ in the norm topology. As a result, we have proved

Claim 3. A sequence $\left(x_{n}\right)$ converges to $x$ in $N$ if and only if $r_{2}\left(x_{n}-x\right) \longrightarrow 0$ as $n \longrightarrow \infty$.

Note also that $r$ and $r_{2}$ coincide on $\operatorname{Ker} f$. Let

$$
S_{2}=\left\{x \in N: r_{2}(x)=1\right\} .
$$

It is easy to see that $h(x)=x / r_{2}(x)$ defines a homeomorphism of $S$ onto $S_{2}$. As invertibility is a topological property, it suffices to show that $S_{2}$ is invertible.

Observe that $f(x)<1$ whenever $x=f(x) x_{0}+y_{x} \in S_{2} \backslash\left\{x_{0}\right\}$ since in this case $r_{2}(x)=\sqrt{f(x)^{2}+r\left(y_{x}\right)^{2}}=1$. This enables us to define a stereographic projection $P: S_{2} \backslash\left\{x_{0}\right\} \longrightarrow \operatorname{Ker} f$ by

$$
P(x)=\frac{y_{x}}{1-f(x)}=\frac{x-f(x) x_{0}}{1-f(x)} .
$$

Claim 4. $P$ is a homeomorphism.

First, we note that for each $x=f(x) x_{0}+y_{x}$ in $S_{2} \backslash\left\{x_{0}\right\}$ with $y_{x}$ in $\operatorname{Ker} f$,

$$
P(x)-x_{0}=\frac{x-f(x) x_{0}}{1-f(x)}-x_{0}=\frac{x-x_{0}}{1-f(x)}
$$

by (2). Therefore,

$$
x=f(x) x_{0}+(1-f(x)) P(x), \quad \forall x \in S_{2} \backslash\left\{x_{0}\right\} .
$$

Thus, $f(x)^{2}+r((1-f(x)) P(x))^{2}=r_{2}(x)^{2}=1$. Since $f(x)<1$, we have

$$
r((1-f(x)) P(x))=(1-f(x)) r(P(x)) .
$$

So $(1-f(x)) r(P(x))^{2}=1+f(x)$, and thus

$$
f(x)=\frac{r(P(x))^{2}-1}{r(P(x))^{2}+1}, \quad \forall x \in S_{2} \backslash\left\{x_{0}\right\} .
$$

Now, suppose $x, x^{\prime}$ in $S_{2} \backslash\left\{x_{0}\right\}$ are such that $P(x)=P\left(x^{\prime}\right)$. Then we have $f(x)=f\left(x^{\prime}\right)$ by (4), and consequently, $x=x^{\prime}$ by (3). In other words, $P$ is one-toone. $P$ is also onto. In fact, for any $y$ in $\operatorname{Ker} f$, we have

$$
P^{-1}(y)=\frac{\left(r(y)^{2}-1\right) x_{0}+2 y}{r(y)^{2}+1}
$$

by (3) and (4) again. The continuity of $P$ and $P^{-1}$ follows from that of $f$ and $r$, respectively.

Claim 5. $S_{2}$ is invertible and the inverting homeomorphisms can be chosen to have period 2.

Let $U$ be a proper open subset in $S_{2}$. Choose an $a$ in $U \backslash\left\{x_{0}\right\}$. There exists a $\delta>0$ such that the closure of $B_{r_{2}, \delta}(a) \cap S_{2}=\left\{x \in S_{2}: r_{2}(x-a)<\delta\right\}$ is contained in $U \backslash\left\{x_{0}\right\}$. Let $b=P(a)$. Since $P$ is an open map, there exists a $\delta^{\prime}>0$ such 
that $B_{r_{2}, \delta^{\prime}}(b) \cap \operatorname{Ker} f=\left\{y \in \operatorname{Ker} f: r_{2}(y-b)<\delta^{\prime}\right\} \subseteq P\left(B_{r_{2}, \delta}(a) \cap S_{2}\right)$. Define the inversion $h_{b, \delta^{\prime}}$ from $\operatorname{Ker} f \backslash\{b\}$ onto itself by the condition that

$$
r_{2}\left(h_{b, \delta^{\prime}}(x)-b\right) r_{2}(x-b)=\delta^{\prime 2}
$$

In other words,

$$
h_{b, \delta^{\prime}}(x)=b+\frac{\delta^{\prime 2}}{r_{2}(x-b)^{2}}(x-b), \quad \forall x \in \operatorname{Ker} f \backslash\{b\} .
$$

Clearly, $h_{b, \delta^{\prime}}=h_{b, \delta^{\prime}}{ }^{-1}$ is continuous and maps $\left\{y \in \operatorname{Ker} f: r_{2}(y-b)>\delta^{\prime}\right\}$ onto $B_{r_{2}, \delta^{\prime}}(b) \cap \operatorname{Ker} f=\left\{y \in \operatorname{Ker} f: r_{2}(y-b)<\delta^{\prime}\right\}$. Define $T: S_{2} \longrightarrow S_{2}$ by

$$
T x= \begin{cases}P^{-1} h_{b, \delta^{\prime}} P(x) & \text { if } x \neq a, x_{0} \\ x_{0} & \text { if } x=a \\ a & \text { if } x=x_{0}\end{cases}
$$

It is plain that $T$ is one-to-one, onto and $T=T^{-1}$. To ensure that $T$ is a homeomorphism, we need only to check the continuity of $T$ at $x_{0}$ and at $a$.

Suppose a sequence $x_{n}=f\left(x_{n}\right) x_{0}+y_{x_{n}}$ in $S_{2} \backslash\left\{x_{0}\right\}$ approaches $x_{0}$. In particular, $1=r_{2}\left(x_{n}\right)^{2}=f\left(x_{n}\right)^{2}+r\left(y_{x_{n}}\right)^{2}$. By (2), we have

$$
r_{2}\left(P\left(x_{n}\right)\right)^{2}=\frac{r\left(y_{x_{n}}\right)^{2}}{\left(1-f\left(x_{n}\right)\right)^{2}}=\frac{1-f\left(x_{n}\right)^{2}}{\left(1-f\left(x_{n}\right)\right)^{2}}=\frac{1+f\left(x_{n}\right)}{1-f\left(x_{n}\right)} \longrightarrow+\infty,
$$

since $f\left(x_{n}\right) \longrightarrow f\left(x_{0}\right)=1$. It then follows from $r_{2}\left(P\left(x_{n}\right)-b\right) \geq r_{2}\left(P\left(x_{n}\right)\right)-$ $r_{2}(b) \longrightarrow+\infty$ that $r_{2}\left(h_{b, \delta^{\prime}} P\left(x_{n}\right)-b\right)=\frac{\delta^{\prime 2}}{r_{2}\left(P\left(x_{n}\right)-b\right)} \longrightarrow 0$ by $(5)$. Hence, $T x_{n}=$ $P^{-1} h_{b, \delta^{\prime}} P\left(x_{n}\right) \longrightarrow P^{-1}(b)=a$ by the continuity of $P^{-1}$. We have thus proved the continuity of $T$ at $x_{0}$. Similarly, suppose a sequence $\left(x_{n}\right)$ in $S_{2} \backslash\left\{x_{0}\right\}$ approaches $a$. Then it follows that $P\left(x_{n}\right) \longrightarrow P(a)=b$. By (5), we have

$$
r_{2}\left(h_{b, \delta^{\prime}} P\left(x_{n}\right)-b\right)=\frac{\delta^{\prime 2}}{r_{2}\left(P\left(x_{n}\right)-b\right)} \longrightarrow+\infty \text {. }
$$

Since

$$
T x_{n}=f\left(T x_{n}\right) x_{0}+\left(1-f\left(T x_{n}\right)\right) P T x_{n}
$$

by (3), we have

$$
1=r_{2}\left(T x_{n}\right)^{2}=f\left(T x_{n}\right)^{2}+\left(1-f\left(T x_{n}\right)\right)^{2} r\left(P T x_{n}\right)^{2} .
$$

Hence, (6) implies that

$$
\sqrt{\frac{1+f\left(T x_{n}\right)}{1-f\left(T x_{n}\right)}}=r\left(P T x_{n}\right)=r\left(h_{b, \delta^{\prime}} P\left(x_{n}\right)\right) \geq r\left(h_{b, \delta^{\prime}} P\left(x_{n}\right)-b\right)-r(-b) \longrightarrow+\infty .
$$

Consequently, $f\left(T x_{n}\right) \longrightarrow 1$ since $f$ is bounded on the norm bounded set $S_{2}$. It then follows from (7) and (8) that

$$
\begin{aligned}
r_{2}\left(T x_{n}-x_{0}\right)^{2} & =\left(f\left(T x_{n}\right)-1\right)^{2}+\left(1-f\left(T x_{n}\right)\right)^{2} r\left(P T x_{n}\right)^{2} \\
& =\left(f\left(T x_{n}\right)-1\right)^{2}+1-f\left(T x_{n}\right)^{2} \longrightarrow 0 .
\end{aligned}
$$

Hence, $T x_{n} \longrightarrow x_{0}$. The continuity of $T$ at $a$ is thus verified. 
Finally, we show that $T\left(S_{2} \backslash U\right) \subseteq U$. If $x_{0} \in S_{2} \backslash U$, then $T x_{0}=a \in U$. If $x \neq x_{0}$ and $x \in S_{2} \backslash U$, then $x$ does not belong to the closure of $B_{r_{2}, \delta}(a) \cap S_{2}$. This implies $P(x)$ does not belong to the closure of $B_{r_{2}, \delta^{\prime}}(b) \cap \operatorname{Ker} f$. In other words, $P(x) \in\left\{y \in \operatorname{Ker} f: r_{2}(y-b)>\delta^{\prime}\right\}$, and thus $h_{b, \delta^{\prime}} P(x) \in B_{r_{2}, \delta^{\prime}}(b) \cap \operatorname{Ker} f \subseteq$ $P\left(B_{r_{2}, \delta}(a) \cap S_{2}\right)$. Consequently, $T x=P^{-1} h_{b, \delta^{\prime}} P(x) \in B_{r_{2}, \delta}(a) \cap S_{2} \subseteq U$. Hence, $T\left(S_{2} \backslash U\right) \subseteq U$, as asserted.

Since $S$ is homeomorphic to $S_{2}$, we conclude that $S$ is invertible. Moreover, the inverting homeomorphisms of $S$ can be chosen to have period 2 as we can do so for the inverting homeomorphisms $T$ of $S_{2}$.

In fact, Theorem 3 also implies Theorem 6 by quoting a deep result of Bessaga and Klee. Recall that the characteristic cone of a convex body $V$ in a topological linear space $X$ is the set $\operatorname{cc} V=\{y \in X$ : there is an $x$ in $X$ with $x+\lambda y \in V$, $\forall \lambda>0\}$. If $\mathrm{cc} V$ is a linear subspace of $X$ of codimension $m(0 \leq m \leq \infty)$, then we say that $V$ has type $m$. $V$ has type $\infty$ also if $\operatorname{cc} V$ is not a linear subspace of $X$. In the following, we write $(X, V) \simeq(Y, U)$ to indicate the existence of a relative homeomorphism from a topological space $X$ onto a topological space $Y$ which sends the topological subspace $V$ of $X$ onto the topological subspace $U$ of $Y$.

Theorem 7 (Bessaga and Klee [2], see also [3, p. 110]). Let $V_{1}$ and $V_{2}$ be closed convex bodies in a topological linear space $X$. Then $\left(X, V_{1}\right) \simeq\left(X, V_{2}\right)$ if and only if $V_{1}$ and $V_{2}$ have the same type. In this case, the topological boundaries of $V_{1}$ and $V_{2}$ are also homeomorphic.

It is evident that all closed bounded convex bodies in a normed space $N$ have the same type, i.e. the dimension of $N$. Therefore, Theorems 3 and 6 imply each other. In fact, much more can be said with the help of Theorem 7 .

Corollary 8. Every infinite-dimensional normed space $N$ is invertible.

Proof. Let $N_{1}=N \times \mathbb{R}$ be the normed space direct product of $N$ and the real line $\mathbb{R}$. Then $N=\left\{x \in N_{1}: f(x)=0\right\}$ for some continuous linear functional $f$ of $N_{1}$. Since the closed half-space $\left\{x \in N_{1}: f(x) \leq 0\right\}$ and the closed unit ball of $N_{1}$ have the same type $(=\infty), N$ is homeomorphic to the unit sphere of $N_{1}$ by Theorem 7 . Consequently, $N$ is invertible.

Remark 9. The invertibility of infinite-dimensional complete normed spaces should not be surprising. Unlike the finite dimensional case, every infinite-dimensional Banach space $E$ is homeomorphic to its unit sphere $S[14,3]$. A key ingredient of the proof is the topological equivalence $L \simeq L \times \mathbb{R}$ for every infinite-dimensional Banach space $L$. The assertion will follow from this since $S$ is homeomorphic to an (infinite-dimensional) closed hyperplane $L$ of $E$ which is in turn homeomorphic to $L \times \mathbb{R} \simeq E$ (see [3, p. 190]). One even has that every infinite-dimensional Hilbert space is real analytically isomorphic to its unit sphere [7]. However, this equivalence between spaces and their unit spheres may not extend to non-complete spaces. In fact, for every infinite-dimensional Banach space $E$ there is a dense linear subspace $L$ of $E$ such that $L$ is not homeomorphic to $L \times \mathbb{R}$ [17]. Consequently, the unit sphere of $L \times \mathbb{R}$, which is homeomorphic to $L$ as in the proof of Corollary 8, is not homeomorphic to the whole space $L \times \mathbb{R}$.

Corollary 10. An infinite-dimensional metrizable locally convex space $X$ is invertible whenever $X$ is complete or $\sigma$-compact. 
Proof. $X$ is homeomorphic to a Hilbert space if $X$ is complete by Theorem 5 , or to a pre-Hilbert space if $X$ is $\sigma$-compact by a result of Bessaga and Dobrowolski [1]. In both cases, $X$ is invertible.

Corollary 11. Every non-empty open convex subset of an invertible topological vector space is invertible. Every closed convex body in an infinite-dimensional Fréchet space or an algebraically $\aleph_{0}$-dimensional normed space is invertible.

Proof. We may assume that $0 \in V$. If $V$ is an open convex subset of a topological vector space $X$, then the map $h(x)=\frac{x}{1-r(x)}$ is a homeomorphism of $V$ onto $X$, where $r$ is the gauge functional of $V$ (see [3, p. 114]). Similarly, $V$ is homeomorphic to the whole space if $V$ is a closed convex body in either an infinite-dimensional Fréchet space (see [3, p. 190]) or an algebraically $\aleph_{0}$-dimensional normed space [5]. In all three cases, $V$ is invertible.

Recall that a subset $A$ of a topological vector space is said to be infinitedimensional if the vector subspace spanned by $A$ is of infinite dimension. The first example of an invertible infinite-dimensional compact set is the Hilbert cube $[0,1]^{\omega}$ given in $[9] .[0,1]^{\omega}$ is the product space of countably infinitely many copies of the compact interval $[0,1]$, and can be embedded into the separable Hilbert space $\ell_{2}$ as the set $\left\{\left(x_{n}\right):\left|x_{n}\right| \leq 1 / n\right\}$. In fact, it was proved in [9] that the product space of arbitrary infinitely many copies of $[0,1]$ is invertible. In a similar manner, one can show that the product space of arbitrary infinitely many copies of the real line $\mathbb{R}$ is also invertible. This turns out to give another proof of the invertibility of infinite-dimensional separable Fréchet spaces, which are known to be homeomorphic to the countable product of lines $\mathbb{R}$ by the Kadec-Anderson Theorem (see $[3$, p. 189]).

Corollary 12. Let $A$ be an infinite-dimensional separable closed convex set in a Fréchet space. $A$ is invertible if and only if $A$ is either compact or not locally compact.

Proof. If $A$ is compact, then $A$ is homeomorphic to the Hilbert cube (see [3, p. 100]). If $A$ is not locally compact, then $A$ is homeomorphic to $\ell_{2}$ [6]. Therefore, $A$ is invertible in both cases. Finally, we note that locally compact invertible space must be compact. Consequently, if $A$ is locally compact but not compact, then $A$ cannot be invertible.

\section{Conjectures}

We do not know too much about the invertibility of the boundary of a closed convex set except for bounded convex bodies (Theorem 6). The following result of Klee might give us some hints.

Proposition 13 (Klee [14]). Suppose $C$ is a closed convex body in an infinitedimensional reflexive Banach space $E$. Then the boundary of $C$ is homeomorphic to $E$ or to $E \times S^{n}$ for some finite $n$.

Concerning Conjecture 4, we collect some results of Henderson which might be useful.

Theorem 14 (Henderson $[11,12])$. Let $H$ be a separable Hilbert space. Every separable metric $H$-manifold $M$ can be embedded as an open subset $U$ of $H$ such that 
the boundary of $U$ and the closure of $U$ are homeomorphic to $U$, and its complement $H \backslash U$ is homeomorphic to $H$.

In the proof of Theorem 1, Doyle and Hocking [8] utilized a high-dimensional Jordan Curve Theorem [4]. In attacking Conjecture 4, we also found that an infinite-dimensional version of Jordan Curve Theorem is needed. We state it as

Conjecture 15. Let $V$ be a connected open subset of an infinite-dimensional Hilbert space $H$. If the boundary of $V$ is homeomorphic to the unit sphere of $H$, then $V$ is homeomorphic to the open unit ball of $H$.

We would like to say a few words to explain why Conjecture 15 is an infinitedimensional extension of the Jordan Curve Theorem. Suppose $V$ is a connected open subset of the plane $\mathbb{R}^{2}$, and the boundary of $V$ is homeomorphic to the unit circle $S^{1}$. Under the usual embedding of $\mathbb{R}^{2}$ into the unit sphere $S^{2}$, we may consider the boundary of $V$ as a homeomorphic image of $S^{1}$ into $S^{2}$. By the Jordan Curve Theorem, this image divides $S^{2}$ into two components each of which is homeomorphic to the open unit ball of $\mathbb{R}^{2}$. By connectedness, $V$ is homeomorphic to one of them. This is also an essential part of Doyle and Hocking's arguments in proving Theorem 1 in $[8]$.

Finally, we would like to express our gratitude to the referee for many useful comments.

\section{REFERENCES}

[1] C. Bessaga and T. Dobrowolski, Affine and homeomorphic embedding into $\ell^{2}$, Proc. Amer. Math. Soc. 125 (1997), 259-268. MR 97e:57022

[2] C. Bessaga and V. L. Klee, Two topological properties of topological linear spaces, Israel J. Math. 2 (1964), 211-220. MR 31:5055

[3] C. Bessaga and A. Pełczyński, Selected Topics in infinite-dimensional topology, Polish Scientific Publishers, Warszawa, 1975. MR 57:17657

[4] M. Brown, A proof of the generalized Schoenflies theorem, Bull. Amer. Math. Soc. 66 (1960), 74-76. MR 22:8470b

[5] H. Corson and V. Klee, Topological classification of convex sets, Proc. Symp. Pure Math. 7 - Convexity, Amer. Math. Soc., Providence, R. I., 1963, 37-51. MR 28:4328

[6] T. Dobrowolski and H. Toruńczyk, Separable complete ANR's admitting a group structure are Hilbert manifolds, Topology and its Applications 12 (1981), 229-235. MR 83a:58007

[7] T. Dobrowolski, Every infinite-dimensional Hilbert space is real-analytically isomorphic with its unit sphere, J. Funct. Analy. 134 (1995), 350-362. MR 96m:46030

[8] P. H. Doyle and J. G. Hocking, A characterization of Euclidean n-spaces, Mich. Math. J., 7 (1960), 199-200. MR 22:12515

[9] , Invertible spaces, Amer. Math. Monthly, 68 (1961), 959-965. MR 24:A1711

[10] W. J. Gray, On the metrizability of invertible spaces, Amer. Math. Monthly 71 (1964), 533534. MR 28:5424

[11] D. W. Henderson, Open subsets of Hilbert space, Compositio Math. 21 (1969), 312-318. MR 40:4975

[12] _ Infinite-dimensional manifolds are open subsets of Hilbert space, Topology 9 (1970), 25-33. MR 40:3581

[13] S. K. Hildebrand and R. L. Poe, The separation axioms for invertible spaces, Amer. Math. Monthly 75 (1968), 391-392. MR 37:2170

[14] V. L. Klee, Topological equivalence of a Banach space with its unit cell, Bull. Amer. Math. Soc. 67 (1961), 286-290. MR 23:A2733

[15] N. Levine, Some remarks on invertible spaces, Amer. Math. Monthly 70 (1963), 181-183. MR 26:4322

[16] P. E. Long, L. L. Herrington, and D. S. Jankovic, Almost-invertible spaces, Bull. Korean Math. Soc. 23 (1986), 91-102. MR 88k:54041 
[17] J. van Mill, Domain invariance in infinite-dimensional linear spaces, Proc. Amer. Math. Soc. 101 (1987), 173-180. MR 88k:57023

[18] S. A. Naimpally, Function spaces of invertible spaces, Amer. Math. Monthly 73 (1966), 513515. MR 33:3269

[19] H. Toruńczyk, Characterizing Hilbert space topology, Fund. Math. CXI (1981), 247-262. MR 82i: 57016

[20] $\longrightarrow$ A correction of two papers concerning Hilbert manifolds, Fund. Math. CXXV (1985), 89-93. MR 87m:57017

Department of Applied Mathematics, National Sun Yat-Sen University, Kaohsiung, 80424, Taiwan, Republic of China

E-mail address: wong@math.nsysu.edu.tw 